\title{
The Functionally Similar to Adaptive Nonlinear Algorithm for Control of Dynamic Plant with Limited Uncertainty
}

\author{
N.D. Polyakhov, A.D. Stotckaia \\ Control Systems Department, Saint-Petersburg electrotechnical university LETI, Saint-Petersburg, Russia
}

\begin{abstract}
A new nonlinear algorithm to control the dynamic plant with limited parametric uncertainty is developed. The algorithm ensures the asymptotic stability and roughness concerning the unaccounted disturbances, and also has almost instantaneous convergence. Simulation studying of operating modes of the synchronous generator with power grid shows the narrow localization (nearly matching) of synchronous generator transient processes in an conditions of significant variations of its parameters with a large multiplicity. Design and implementation of these algorithms is quite simple and does not require precise debugging.
\end{abstract}

\section{Introduction}

There are many methods of dynamic plants with uncertainty control, including adaptive algorithms that continuously held a leading position. For constructing an adaptive tuning algorithm for controller parameters the method of Lyapunov functions and a variety of gradient methods are frequently used [1].

The core of these algorithms are algorithms in the form of differential equations $\dot{k}=\Phi(e, x)$, where $\mathrm{k}$ matrix of adjustable parameters, the right side of the equations are defined during the synthesis. Such algorithms include in their structure integrators for updating the configurable parameters. This makes them fundamentally inertia and structurally unstable to unaccounted perturbations because of the presence of stability only in the ideal case of decisions (without disturbances), but in conditions of disturbances action rising drift parameters is appeared.

To eliminate these phenomena, that is, to achieve roughness (robustness) of convergence, algorithms are modified by the introduction of feedback by adjustable parameters (regularization). As a result the algorithms of the form $\dot{k}=\Phi(e, x)-\alpha k$ ( $\alpha-$ feedback depth), in which there is an exponential (dissipative) adaptive processes, can be obtained. However, the use of such means for achieving algorithm roughness is associated with the availability of permanent transient processes in the adaptive system: error vector $e$ must not be zero.

In order to relax this condition it is necessary to strengthen the parameter $\alpha$ and increase the speed setting that in the multidimensional case is restrictively on stability. In any case it is technically unacceptable.

The paper presents synthesis of a new algorithm in a form $k=F(e, x)$. For its construction Lyapunov functions method is also used. The convergence of processes is almost instantaneous and has an asymptotic nature. Structurally, it looks like a nonlinear algorithm such as parameter feedback, but functionally is efficient with significant (multiple) deviations of the dynamic parameters of control plant.

\section{Theoretical results}

\subsection{Introductory Notes}

Assume that the control plant is specified as:

$$
\begin{gathered}
\dot{x}(t)=A x(t)+B u(t), \\
x\left(t_{0}\right)=x_{0}
\end{gathered}
$$

where $x(t)-$ state vector, $u(t)=u^{a}(t)+g(t)-$ nonlinear control vector, $g(t)$ - input influences vector.

Matrix shown in (1) are described as follows:

$B=\left\{b_{i j}\right\}_{n \times m}-$ matrix with known elements;

$A=\left\{a_{i j}\right\}_{n \times n}-$ matrix with interval type of uncertainty $\theta$;

$\theta=\left\{\theta \in R^{l}:\left|\theta_{i}-\theta_{i}^{*}\right| \leq \Delta \theta_{i}, i=\overline{1, l}\right\}, l=n^{2}$

$\theta=\left[a_{11}, a_{12}, a_{13}, \ldots, a_{n n}\right]$

$\theta_{i}^{*}$ - rated value.

Construction of the algorithm is represented by the scheme of the system with a reference model [2].

Let us suppose that the equation of reference model is given by:

$$
\dot{\hat{x}}(t)=A_{0} \hat{x}(t)+B_{0} g(t)
$$


where $\hat{x}(t)-n$-vector of reference model, $A_{0}, B_{0}-$ reference model matrix corresponding to the desired dynamics.

Let us introduce the control error:

$$
e_{i}(t)=x_{i}(t)-\hat{x}_{i}(t), i=\overline{1, n}
$$

On the basis of the expressions (1), (2) after several transformations we get the following equation:

$$
\begin{gathered}
\dot{e}(t)=A e(t)+B u^{a}(t)+\delta, \\
e\left(t_{0}\right)=e_{0}, \\
\delta(t)=\left[\left(A-A_{0}\right) \hat{x}(t)+\left(B-B_{0}\right) g(t)\right] .
\end{gathered}
$$

For the case when the matrix B is irreversible, modifying the equation (3), so that

$$
\dot{e}(t)=A e(t)+B u^{a}(t)+B B^{+}+\delta(t) .
$$

Performing alignment condition

$$
\lim _{t \rightarrow \infty}\left(B B^{+} \delta(t)-\delta(t)\right)=0
$$

it leads to the equivalence of the equation (3) and the modified equation.

\subsection{Lemma}

The control law $u^{a}(t)$ ensures asymptotic stability of the trivial solution of the equation (3) if and only if it is provided by the condition $\lim _{t \rightarrow \infty} \delta(t)=0$.

Indeed, the achievement of an asymptotically stable zero solution e $(\mathrm{t})=0$ is associated with the provision of control low $u^{a}(t)$ the condition $A \stackrel{u^{a}(t)}{\longrightarrow} A_{0}$, with the result that, as well as in the choice $B=B_{0}$, we obtain conclusion of the lemma.

Consider the equation (3), when $\delta(t)=0$, for determining the control structure:

$$
\dot{e}(t)=A e(t)+B u^{a}(t), e\left(t_{0}\right)=e_{0}
$$

Let us suppose that the controller description has the form of a feedback:

$$
\begin{gathered}
u^{a}(t)=K e(t), \\
K=\{k i j\}_{m \times n}
\end{gathered}
$$

where $K$-adjustable parameters matrix.

Let us define elements of adjustable parameters matrix $K(t)$ for the asymptotic stability of the system (1). Suppose that Lyapunov function is in the form [1]:

$$
J(t)=\frac{1}{2} e^{T}(t) e(t)
$$

then:

$$
\Psi(t)=\dot{J}(t)=e^{T}(t) \dot{e}(t) .
$$

To ensure the asymptotic stability of system (5) is sufficient to:

$$
\Psi(t)<0
$$

From the expressions (5) and (6) following equations are obtained:

$$
\dot{e}(t)=\Gamma(t) e(t), \Gamma(t)=(A+B K) .
$$

Then, by the last equation:

$$
\Psi(t)=e^{T}(t) \Gamma(t) e(t)=\sum_{i=1}^{n} e_{i i}^{2} \gamma_{i i}+\sum_{\substack{i, j=1 \\ i \neq j}}^{n} \gamma_{i j} e_{i} e_{j} .
$$

\subsection{Lemma}

Let us assume that

$$
\begin{gathered}
\Psi(t)=\Psi_{1}(t)+\Psi_{2}(t) \oplus \\
\Psi_{1}(t)=\sum_{i=1}^{n} \gamma_{i i} e_{i i}^{2} \quad \Psi_{2}(t)<0, i=\overline{1, n}
\end{gathered}
$$

Then, if

$$
\Psi_{1}(t)<0, \Psi_{2}(t)<0, i=\overline{1, n}
$$

the condition of asymptotic stability (6) is satisfied.

Let us take the diagonal elements of $\Gamma(t)$ are constant and negative, that is $\gamma_{i i}<0$ and then $\Psi_{1}(t)<0$ and find the conditions for the inequality $\Psi_{2}(t)<0$. Use the substitution [3]:

$$
\gamma_{i j}(t)=\alpha_{i j}{ }^{-1} e_{i}(t) e_{j}(t), \quad i, j=1, n \quad i \neq j
$$

hence,

$$
\Psi_{2}(t)=\sum_{\substack{i, j=1 \\ i \neq j}}^{n} \gamma_{i j} e_{i} e_{j}=\sum_{\substack{i, j=1 \\ i \neq j}}^{n} \alpha_{i j} \gamma_{i j}^{2}(t)
$$

Thus, from (9) it follows that if $\alpha_{i j}<0$, then $\Psi_{2}(t)<0$ and if $\gamma_{i i}<0$ and constant $\alpha_{i j}<0, i \neq j$, then the asymptotic stability condition (7) of the system (5) is satisfied.

From the expression (8) we obtain the matrix of adjustable parameters $K(t)$ ), that has the following form:

$$
K(t)=B^{-1}(\Gamma-A),
$$

if $n=m$ and $B$ has an inverse matrix; when $n \neq m$ matrix $B^{-1}$ is replaced by the pseudoinverse $B^{+}$.

For the calculation of matrix $K(t)$ "nominal" values of elements $\theta_{i}{ }^{*}$ of the matrix A are used.

\subsection{Theorem}

The system (1) has:

1) asymptotic stability of processes for e (t) with control law $u^{a}(t)=K e(t), K(t)=B^{-1}(\Gamma-A) u$ $K(t)=B^{+}(\Gamma-A)$, when the alignment condition (4) performs,

2) dissipative processes on the condition of not doing (4), and limitations of all the variables of the system state, but with a regulation radius limit set by selecting parameters of the control law.

\section{An example of algorithm using}

As a dynamic object with bounded uncertainties, consider a synchronous generator (SG), which is described in certain assumptions equations system deviation $[4,5]$ 
$\Delta \dot{E}_{q}(t)=-\frac{1}{k_{3} T_{d 0}} \Delta E_{q}(t)-\frac{k_{4}}{T_{d 0}} \Delta \delta(t)+\frac{1}{T_{d 0}} \Delta E_{f}(t)$,

$\Delta \dot{\omega}(t)=-\frac{k_{2}}{T_{j}} \Delta E_{q}(t)-\frac{k_{1}}{T_{j}} \Delta \delta(t)+\frac{1}{T_{j}} \Delta M_{M}(t)$,

$\Delta \dot{\delta}(t)=\Delta \omega(t)$,

$\Delta U(t)=k_{5} \Delta \delta(t)+k_{6} \Delta E_{q}(t)$,

where $\Delta U(t), \Delta \delta(t), \Delta E_{q}(t), \Delta \omega(t)$-respectively, voltage deviation, load angle, emf (electric motive force) and the angular frequency; $\Delta M_{\mathrm{M}}(t)-$ of turbine mechanical torque deviation, $\Delta E_{f}(t)-$ the emf deviation of exciter.

Here, $k_{1}-$ a coefficient characterizing the change in the electrical power when changing the angle of the rotor, ie, synchronizing torque; $k_{2}-$ a coefficient characterizing the change in the electrical power when changing the conditions of constant flux in the rotor angle; $k_{3}-$ a coefficient characterizing the influence of the external resistance, $\boldsymbol{k}_{4}-$ a coefficient characterizing the demagnetizing effect of changing the angle of the rotor; $k_{5}-$ a coefficient characterizing the change in voltage on the tires when the generator rotor angle change in conditions of constant emf; $k_{6}-$ a coefficient characterizing the voltage on the generator, when emf changes in conditions of constant angle.

The study adopted the equivalent system of equations of the object, characterized by the presence of the right sides of the brackets, that can change the sign at parameter variation. This property characterizes the uncertainty in a more complicated and is therefore attractive for the study.

Thus, research equations are

$$
\begin{gathered}
\Delta \dot{U}(t)=-\frac{1}{k_{3} \tau_{d}} \Delta U(t)+k_{5} \Delta \omega(t)+\left(\frac{k_{5}}{k_{3} \tau_{d}}-\frac{k_{6} k_{4}}{\tau_{d}}\right) \Delta \delta(t)+\frac{k_{6}}{\tau_{d}} \Delta E_{f d}(t), \\
\Delta \dot{\omega}(t)=-\frac{k_{2}}{k_{6} T_{j}} \Delta U(t)+\left(\frac{k_{2} k_{5}}{k_{6} \tau_{j}}-\frac{k_{1}}{T_{j}}\right) \Delta \delta(t)+\frac{1}{T_{j}} \Delta M_{M}(t), \\
\Delta \dot{\delta}(t)=\Delta \omega(t) .
\end{gathered}
$$

Simulation of synchronous generator control systems with a nonlinear controller is done in MATLAB/Simulink environment for the parameters of synchronous generator and the reference model listed in Table 1.

Table 1. Parameters of synchronous generator and the reference model

\begin{tabular}{|c|c|c|}
\hline Parameters & $\begin{array}{c}\text { Synchronous } \\
\text { generator }\end{array}$ & $\begin{array}{c}\text { Reference } \\
\text { model }\end{array}$ \\
\hline$k_{1}$ & 1,773 & 10,9 \\
\hline$k_{2}$ & 0,854 & 2,7435 \\
\hline$k_{3}$ & 0,346 & 0,13 \\
\hline$k_{4}$ & 1,49 & 0,4655 \\
\hline$k_{5}$ & 0,038 & 0,1 \\
\hline$k_{6}$ & 0,584 & 1,9512 \\
\hline
\end{tabular}

\begin{tabular}{|c|c|c|}
\hline Parameters & $\begin{array}{c}\text { Synchronous } \\
\text { generator }\end{array}$ & $\begin{array}{c}\text { Reference } \\
\text { model }\end{array}$ \\
\hline$\tau_{\mathrm{d}}, \mathrm{s}$ & 4 & 4 \\
\hline$T_{j}, \mathrm{~s}$ & 2,5 & 2,5 \\
\hline
\end{tabular}

As a result, obtained the following:

$$
\begin{gathered}
A=\left[\begin{array}{ccc}
-0,723 & 0,038 & -0,19 \\
-0,585 & 0 & 0.687 \\
0 & 1 & 0
\end{array}\right] \\
B=\left[\begin{array}{cc}
0,146 & 0 \\
0 & 0,4 \\
0 & 0
\end{array}\right] \\
B^{+}=\left[\begin{array}{ccc}
6,85 & 0 & 0 \\
0 & 2,5 & 0
\end{array}\right] .
\end{gathered}
$$

Nominal values of the elements of the matrix $A$, taking into account peculiarities of the model of an oscillator (change in sign of the difference in brackets) and the presence of uncertainty, are chosen as follows:

$$
a_{i j}^{*}=\left[\begin{array}{lllllll}
-0,723 & 0,038 & -0,19 ;-0,585 & 0 & 5 ; 0 & 1 & 0
\end{array}\right]
$$

For the "good" quality of control processes the following nonlinear controller parameters control are chosen:

$$
\alpha_{12}=-10, \alpha_{13}=-3, \alpha_{21}=-6, \alpha_{23}=-4 \text {. }
$$

In addition, the components $k_{11}, k_{22}$ are selected arbitrarily in the simulation process (but with negative values): $k_{11}=-18, k_{22}=-8$. Then a nonlinear algorithm in accordance to (10) has the following form:

$$
\begin{gathered}
k_{12}=6,85\left(-10^{-1} e_{1} e_{2}-0,038\right), \\
k_{13}=6,85\left(-3^{-1} e_{1} e_{3}+0,19\right), \\
k_{21}=2,5\left(-6^{-1} e_{2} e_{1}+0,585\right), \\
k_{23}=2,5\left(-4^{-1} e_{2} e_{3}-5\right) .
\end{gathered}
$$

The equations of nonlinear control (6) is obtained in the form:

$$
\begin{gathered}
u_{1}^{a}(t)=k_{11} e_{1}(t)+k_{12} e_{2}(t)+k_{13} e_{3}(t) \\
u_{2}^{a}(t)=k_{21} e_{1}(t)+k_{22} e_{2}(t)+k_{23} e_{3}(t) \\
u^{a}(t)=\left[u_{1}^{a}(t) u_{2}^{a}(t)\right]^{T}=\left[\Delta E_{f d} \Delta M_{M}(t)\right]^{T}, \\
e_{1}(t)=\Delta U(t), e_{2}(t)=\Delta \omega(t), e_{3}(t)=\Delta \delta(t) .
\end{gathered}
$$

The efficiency of nonlinear algorithm was investigated under the following conditions:

- changing a few values of elements of matrix $A$ (synchronous generator);

- considering of the transient processes of voltage and frequency.

The values of coefficients simultaneously increase or decrease in 5 times. Then:

$$
A_{1}=\left[\begin{array}{ccc}
-0,723 & 0,19 & -0,19 \\
-2,925 & 0 & 3,435 \\
0 & 1 & 0
\end{array}\right]
$$


or

$$
A_{2}=\left[\begin{array}{ccc}
-0,723 & 0,0076 & -0,19 \\
-0,117 & 0 & 0,137 \\
0 & 1 & 0
\end{array}\right] .
$$

The simulation results of synchronous generator transient processes with three sets of matrix elements (reduction/increasing 5 times and the nominal values) in MatLab/Simulink environment shown in Fig. 1 an Fig. 2.

Fig. 1 and Fig. 2 show transient processes of the synchronous generator without adaptive control and under adaptive control. Based on the simulation results, it is clear that the transient response of synchronous generator with controller (the transient time: $3.5-4.5$ seconds) are located in the vicinity of a sufficiently narrow curves.

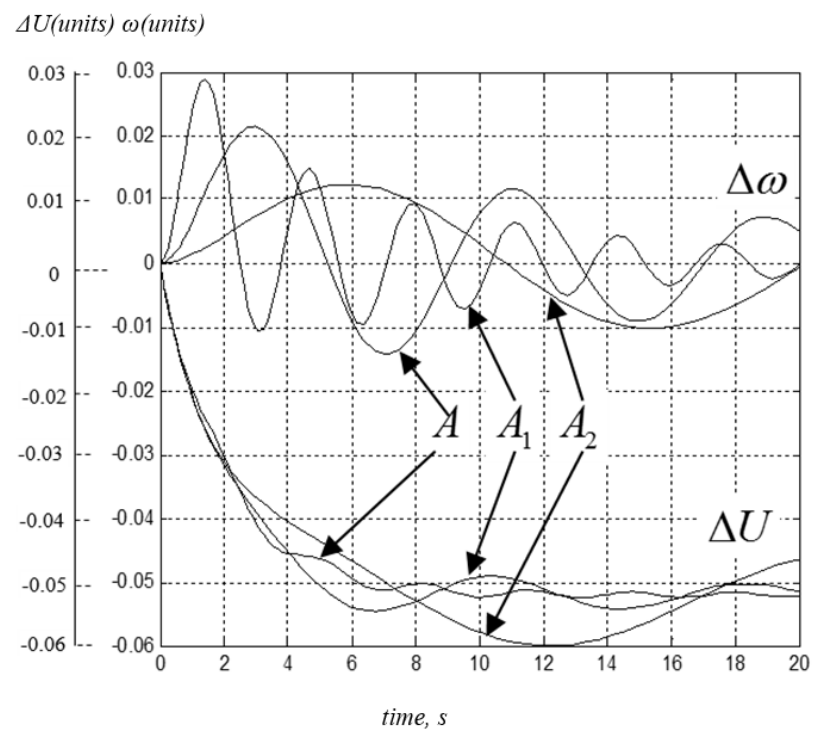

Figure 1. Transient processes of the synchronous generator without adaptive control

$\Delta U$ (units) $\omega$ (units)

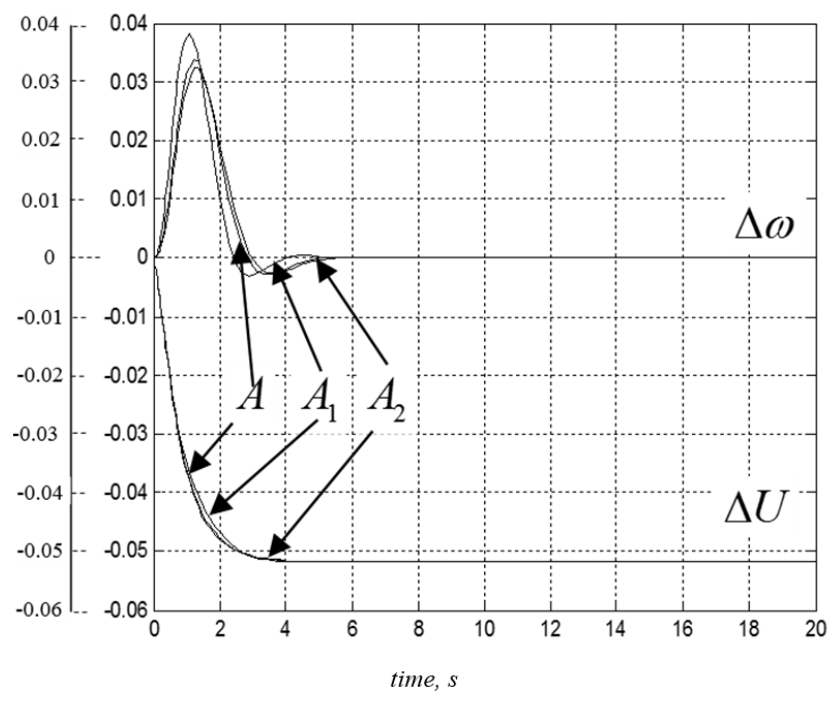

Figure 2. Transient processes of the synchronous generator under adaptive control
The development of automatic excitation controllers are the basic methods of linear control theory in the synthesis of control algorithms, and used only the standard PD - laws. However, in control problems with the uncertainty of a synchronous generator (parametric perturbations), these methods are ineffective.

To stabilize the dynamic behavior of synchronous generator excitation system in different operation conditions most appropriate introduction of adaptive control systems based on models and inertia less algorithms.

The deficiency of adaptive algorithms in schemes with reference / adjustable model and parametric adaptation is that the convergence of the algorithm is exponentially stable in the variables error, but the parametric adaptation processes by Lyapunov indicates unrobust stability convergence parametric adaptation processes.

Synthesized law fast-response parameter adaptation has asymptotic (here exponential) convergence process with zero adaptation time, a robust unaccounted disturbances and large parametric deviations. System simulation study shows effective treatment to parametric deviations even with a change of its sign.

The mode of bifurcation occurrence [6] shows the ability to limit the size of the attractor and even its complete suppression. The algorithm is preferable for practical use, as freely and universally implemented for controlling technical objects.

\section{Acknowledgment}

The research work was partly supported by basic part of Russian Ministry state task (project number 2014/187 the implementation of government task in the sphere of scientific activity).

\section{References}

1. A. L. Fradkov, "Adaptive control in complex systems: searchless methods" M: Nauka, 1990, 296 p.

2. Yu. A. Bortsov, N. D. Polyakhov, V. V. Putov "Electromechanical systems with adaptive and modal control" L: Energoatomisdat, 1984. 216 p.

3. G. A. Kozhekova "The calculation of the adaptive control systems for synchronous generator" Izvestia KSTU im. I. Razzakova, \# 21, 2010.

4. P. Anderson, A. Fuad "Power systems control and sustainability" M: Energia, 1980.

5. N. D. Polyakhov, Ha Anh Tuan Adaptive "Control of synchronous generator based on inertialess parametric adaptation algorithm" // Journal "Electrichestvo", 2014. Vol. 12. - p.47-52

6. N. D. Polyakhov, Ha Anh Tuan "Adaptive control of a synchronous generator in the mode of bifurcation appearance"// Online Journal "Naukovedenie", 2014. Issue 5.

\section{Conclusion}

